\title{
A Numerical Approach to Solving an Inverse Heat Conduction Problem Using the Levenberg-Marquardt Algorithm
}

\author{
Tao Min, ${ }^{1,2}$ Xing Chen, ${ }^{1}$ Yao Sun, ${ }^{1}$ and Qiang Huang ${ }^{2}$ \\ ${ }^{1}$ School of Science, Xian University of Technology, Xian, Shaanxi 710054, China \\ ${ }^{2}$ State Key Laboratory of Eco-Hydraulic Engineering in Shaanxi, Xian University of Technology, Xian, Shaanxi 710048, China \\ Correspondence should be addressed to Tao Min; mintao@xaut.edu.cn
}

Received 14 April 2014; Accepted 8 June 2014; Published 24 June 2014

Academic Editor: Hua Fan

Copyright (c) 2014 Tao Min et al. This is an open access article distributed under the Creative Commons Attribution License, which permits unrestricted use, distribution, and reproduction in any medium, provided the original work is properly cited.

\begin{abstract}
This paper is intended to provide a numerical algorithm involving the combined use of the Levenberg-Marquardt algorithm and the Galerkin finite element method for estimating the diffusion coefficient in an inverse heat conduction problem (IHCP). In the present study, the functional form of the diffusion coefficient is unknown a priori. The unknown diffusion coefficient is approximated by the polynomial form and the present numerical algorithm is employed to find the solution. Numerical experiments are presented to show the efficiency of the proposed method.
\end{abstract}

\section{Introduction}

The numerical solution of the inverse heat conduction problem (IHCP) requires determining diffusion coefficient from additional information. Inverse heat conduction problems have many applications in various branches of science and engineering; mechanical and chemical engineers, mathematicians, and specialists in many other science branches are interested in inverse problems, each with different application in mind [1-15].

In this work, we propose an algorithm for numerical solving of an inverse heat conduction problem. The algorithm is based on the Galerkin finite element method and LevenbergMarquardt algorithm $[16,17]$ in conjunction with the leastsquares scheme. It is assumed that no prior information is available on the functional form of the unknown diffusion coefficient in the present study; thus, it is classified as the function estimation in inverse calculation. Run the numerical algorithm to solve the unknown diffusion coefficient which is approximated by the polynomial form. The LevenbergMarquardt optimization is adopted to modify the estimated values.

The plan of this paper is as follows. In Section 2, we formulate a one-dimensional IHCP. In Section 3, the numerical algorithm is derived. Calculation of sensitivity coefficients will be discussed in Section 4. In order to discuss some numerical aspects, two examples are given in Section 5. Section 6 ends this paper with a brief discussion on some numerical aspects.

\section{Description of the Problem}

The mathematical formulation of a one-dimensional heat conduction problem is given as follows:

$$
\begin{array}{r}
\frac{\partial u}{\partial t}=\frac{\partial}{\partial x}\left[q(x) \frac{\partial u}{\partial x}\right]+f(x, t), \\
(x, t) \in(0, L) \times(0, T],
\end{array}
$$

with the initial condition

$$
u(x, 0)=u_{0}(x), \quad 0 \leq x \leq L,
$$

and Dirichlet boundary conditions

$$
\begin{array}{ll}
u(0, t)=g_{1}(t), & 0 \leq t \leq T, \\
u(1, t)=g_{2}(t), & 0 \leq t \leq T,
\end{array}
$$

where $f(x, t), u_{0}(x), g_{1}(t), g_{2}(t)$, and $q(x)$ are continuous known functions. We consider the problem (1)-(3) as a direct 
problem. As we all know, if $u_{0}(x), g_{1}(t), g_{2}(t)$ are continuous functions and $q(x)$ is known, the problem (1)-(3) has a unique solution.

For the inverse problem, the diffusion coefficient $q(x)$ is regarded as being unknown. In addition, an overspecified condition is also considered available. To estimate the unknown coefficient $q(x)$, the additional information on the boundary $x=x_{0}, 0<x_{0}<L$, is required. Let the $u(x, t)$ taken at $x=x_{0}$ over the time period $[0, T]$ be denoted by

$$
u\left(x_{0}, t\right)=g(t), \quad 0 \leq t \leq T .
$$

It is evident that for an unknown function $q(x)$, the problem (1)-(3) is underdetermined and we are forced to impose additional information (4) to provide a unique solution pair $(u(x, t), q(x))$ to the inverse problem (1)-(4).

We note that the measured overspecified condition $u\left(x_{0}, t\right)=g(t)$ should contain measurement errors. Therefore, the inverse problem can be stated as follows: by utilizing the above-mentioned measured data, estimate the unknown function $q(x)$.

In this work, the polynomial form is proposed for the unknown function $q(x)$ before performing the inverse calculation. Therefore, $q(x)$ is approximated as

$$
\begin{aligned}
q(x) & \approx q(x) \\
= & p_{1}+p_{2} x+p_{3} x^{2}+\cdots+p_{m+1} x^{m},
\end{aligned}
$$

where $p_{1}, p_{2}, \ldots, p_{m+1}$ are constants which remain to be determined simultaneously. The unknown coefficients $p_{1}, p_{2}, \ldots, p_{m+1}$ can be determined by using least-squares method. The error in the estimate

$$
\begin{aligned}
F & \left(p_{1}, p_{2}, \ldots, p_{m+1}\right) \\
& =\sum_{i=1}^{n}\left[u\left(x_{0}, t_{i}, p_{1}, p_{2}, \ldots, p_{m+1}\right)-g\left(t_{i}\right)\right]^{2}
\end{aligned}
$$

is to be minimized. Here, $u\left(x_{0}, t_{i}, p_{1}, p_{2}, \ldots, p_{m+1}\right)$ are the calculated results. These quantities are determined from the solution of the direct problem which is given previously by using an approximated $q \hat{(x)}$ for the exact $q(x)$. The estimated values of $p_{j}, j=1,2, \ldots, m+1$, are determined until the value of $F\left(p_{1}, p_{2}, \ldots, p_{m+1}\right)$ is minimum. Such a norm can be written as

$$
F(\mathbf{P})=[\mathbf{U}(\mathbf{P})-\mathbf{G}]^{T}[\mathbf{U}(\mathbf{P})-\mathbf{G}],
$$

where $\mathbf{P}^{T}=\left[p_{1}, p_{2}, \ldots, p_{m+1}\right]$ denotes the vector of unknown parameters and the superscript $T$ above denotes transpose. The vector $[\mathbf{U}(\mathbf{P})-\mathbf{G}]^{T}$ is given by

$$
\begin{aligned}
{[\mathbf{U}(\mathbf{P})-\mathbf{G}]^{T} } \\
=\left[u\left(x_{0}, t_{1}, \mathbf{P}\right)-g\left(t_{1}\right), u\left(x_{0}, t_{2}, \mathbf{P}\right)\right. \\
\left.\quad-g\left(t_{2}\right), \ldots, u\left(x_{0}, t_{n}, \mathbf{P}\right)-g\left(t_{n}\right)\right] .
\end{aligned}
$$

$F(\mathbf{P})$ is real-valued bounded function defined on a closed bounded domain $D \subset R^{m+1}$. The function $F(\mathbf{P})$ may have many local minima in $D$, but it has only one global minimum. When $F(\mathbf{P})$ and $D$ have some attractive properties, for instance, $F(\mathbf{P})$ being a differentiable concave function and $D$ being a convex region, then a local maximum problem can be solved explicitly by mathematical programming methods.

\section{Overview of the Levenberg-Marquardt Method}

The Levenberg-Marquardt method, originally devised for application to nonlinear parameter estimation problems, has also been successfully applied to the solution of linear illconditioned problems. Such a method was first derived by Levenberg (1944) by modifying the ordinary least-squares norm. Later Marquardt (1963) derived basically the same technique by using a different approach. Marquardt's intention was to obtain a method that would tend to the Gauss method in the neighborhood of the minimum of the ordinary least-squares norm and would tend to the steepest descent method in the neighborhood of the initial guess used for the iterative procedure.

To minimize the least-squares norm (7), we need to equate to zero the derivatives of $F(\mathbf{P})$ with respect to each of the unknown parameters $\left[p_{1}, p_{2}, \ldots, p_{m+1}\right]$; that is,

$$
\frac{\partial F(\mathbf{P})}{\partial p_{1}}=\frac{\partial F(\mathbf{P})}{\partial p_{2}}=\cdots=\frac{\partial F(\mathbf{P})}{\partial p_{m+1}}=0 .
$$

Let us introduce the sensitivity or Jacobian matrix, as follows:

$$
\begin{aligned}
\mathbf{J}(\mathbf{P}) & =\left[\frac{\partial \mathbf{U}^{T}(\mathbf{P})}{\partial \mathbf{P}}\right]^{T} \\
& =\left[\begin{array}{cccc}
u_{p_{1}}\left(x_{0}, t_{1}, \mathbf{P}\right) & u_{p_{2}}\left(x_{0}, t_{1}, \mathbf{P}\right) & \cdots & u_{p_{m+1}}\left(x_{0}, t_{1}, \mathbf{P}\right) \\
u_{p_{1}}\left(x_{0}, t_{2}, \mathbf{P}\right) & u_{p_{2}}\left(x_{0}, t_{2}, \mathbf{P}\right) & \cdots & u_{p_{m+1}}\left(x_{0}, t_{2}, \mathbf{P}\right) \\
\vdots & \vdots & \cdots & \vdots \\
u_{p_{1}}\left(x_{0}, t_{n}, \mathbf{P}\right) & u_{p_{2}}\left(x_{0}, t_{n}, \mathbf{P}\right) & \cdots & u_{p_{m+1}}\left(x_{0}, t_{n}, \mathbf{P}\right)
\end{array}\right],
\end{aligned}
$$

or

$$
\begin{aligned}
& J_{i j}=u_{p_{j}}\left(x_{0}, t_{i}, \mathbf{P}\right)=\frac{\partial u\left(x_{0}, t_{i}, \mathbf{P}\right)}{\partial p_{j}}, \\
& i=1,2, \ldots, n, \quad j=1,2, \ldots, m+1 .
\end{aligned}
$$

The elements of the sensitivity matrix are called the sensitivity coefficients, and the results of differentiation (9) can be written down as follows:

$$
-2 \mathbf{J}^{T}(\mathbf{P})[\mathbf{U}(\mathbf{P})-\mathbf{G}]=0 .
$$

For linear inverse problem, the sensitivity matrix is not a function of the unknown parameters. Equation (12) can be solved then in explicit form as follows:

$$
\mathbf{P}=\left(\mathbf{J}^{T} \mathbf{J}\right)^{-1} \mathbf{J}^{T} \mathbf{G}
$$

In the case of a nonlinear inverse problem, the matrix $\mathbf{J}$ has some functional dependence on the vector $\mathbf{P}$. The solution 
of (12) requires an iterative procedure, which is obtained by linearizing the vector $\mathbf{U}(\mathbf{P})$ with a Taylor series expansion around the current solution at iteration $k$. Such a linearization is given by

$$
\mathbf{U}(\mathbf{P})=\mathbf{U}\left(\mathbf{P}^{k}\right)+\mathbf{J}^{k}\left(\mathbf{P}-\mathbf{P}^{k}\right)
$$

where $\mathbf{U}\left(\mathbf{P}^{k}\right)$ and $\mathbf{J}^{k}$ are the estimated temperatures and the sensitivity matrix evaluated at iteration $k$, respectively. Equation (14) is substituted into (13) and the resulting expression is rearranged to yield the following iterative procedure to obtain the vector of unknown parameters $\mathbf{P}$ :

$$
\mathbf{P}^{k+1}=\mathbf{P}^{k}+\left[\left(\mathbf{J}^{k}\right)^{T} \mathbf{J}^{k}\right]^{-1}\left(\mathbf{J}^{k}\right)^{T}\left[\mathbf{G}-\mathbf{U}\left(\mathbf{P}^{k}\right)\right]
$$

The iterative procedure given by (15) is called the Gauss method. Such method is actually an approximation for the Newton (or Newton-Raphson) method. We note that (13) and the implementation of the iterative procedure given by (15) require the matrix $\mathbf{J}^{T} \mathbf{J}$ to be nonsingular, or

$$
\left|\mathbf{J}^{T} \mathbf{J}\right| \neq 0
$$

where $|\cdot|$ is the determinant.

Formula (16) gives the so-called identifiability condition; that is, if the determinant of $\mathbf{J}^{T} \mathbf{J}$ is zero, or even very small, the parameters $p_{j}$, for $j=1,2, \ldots, m+1$, cannot be determined by using the iterative procedure of (15).

Problems satisfying $\left|\mathbf{J}^{T} \mathbf{J}\right| \approx 0$ are denoted as illconditioned. Inverse heat transfer problems are generally very ill-conditioned, especially near the initial guess used for the unknown parameters, creating difficulties in the application of (13) or (15). The Levenberg-Marquardt method alleviates such difficulties by utilizing an iterative procedure in the form

$$
\mathbf{P}^{k+1}=\mathbf{P}^{k}+\left[\left(\mathbf{J}^{k}\right)^{T} \mathbf{J}^{k}+\mu^{k} \Omega^{k}\right]^{-1}\left(\mathbf{J}^{k}\right)^{T}\left[\mathbf{G}-\mathbf{U}\left(\mathbf{P}^{k}\right)\right]
$$

where $\mu^{k}$ is a positive scalar named damping parameter and $\Omega^{k}$ is a diagonal matrix.

The purpose of the matrix term $\mu^{k} \Omega^{k}$ is to damp oscillations and instabilities due to the ill-conditioned character of the problem, by making its components large as compared to those of $\mathbf{J}^{T} \mathbf{J}$ if necessary. $\mu^{k}$ is made large in the beginning of the iterations, since the problem is generally ill-conditioned in the region around the initial guess used for iterative procedure, which can be quite far from the exact parameters. With such an approach, the matrix $\mathbf{J}^{T} \mathbf{J}$ is not required to be nonsingular in the beginning of iterations and the LevenbergMarquardt method tends to the steepest descent method; that is, a very small step is taken in the negative gradient direction. The parameter $\mu^{k}$ is then gradually reduced as the iteration procedure advances to the solution of the parameter estimation problem, and then the LevenbergMarquardt method tends to the Gauss method given by (15). The following criteria were suggested in literature [13] to stop the iterative procedure of the Levenberg-Marquardt method given by (17):

$$
\begin{gathered}
F\left(\mathbf{P}^{k+1}\right)<\varepsilon_{1}, \\
\left\|\left(\mathbf{J}^{k}\right)\left[\mathbf{G}-\mathbf{U}\left(\mathbf{P}^{k}\right)\right]\right\|<\varepsilon_{2}, \\
\left\|\mathbf{P}^{k+1}-\mathbf{P}^{k}\right\|<\varepsilon_{3},
\end{gathered}
$$

where $\varepsilon_{1}, \varepsilon_{2}$, and $\varepsilon_{3}$ are user prescribed tolerances and $\|\cdot\|$ denotes the Euclidean norm. The criterion given by (18) tests if the least-squares norm is sufficiently small, which is expected in the neighborhood of the solution for the problem. Similarly, (19) checks if the norm of the gradient of $F(\mathbf{P})$ is sufficiently small, since it is expected to vanish at the point where $F(\mathbf{P})$ is minimum. The last criterion given by (20) results from the fact that changes in the vector of parameters are very small when the method has converged. Generally, these three stopping criteria need to be tested and the iterative procedure of the Levenberg-Marquardt method is stopped if any of them is satisfied.

Different versions of the Levenberg-Marquardt method can be found in the literature, depending on the choice of the diagonal matrix $\Omega^{k}$ and on the form chosen for the variation of the damping parameter $\mu^{k}$. In this paper, we choose $\Omega^{k}$ as

$$
\Omega^{k}=\operatorname{diag}\left[\left(\mathbf{J}^{k}\right)^{T} \mathbf{J}^{k}\right]
$$

Suppose that the vectors of temperature measurements $\mathbf{G}=$ $\left[g\left(t_{1}\right), g\left(t_{2}\right), \ldots, g\left(t_{n}\right)\right]$ are given at times $t_{i}, i=1,2, \ldots, n$, and an initial guess $\mathbf{P}^{0}$ is available for the vector of unknown parameters $\mathbf{P}$. Choose a value for $\mu^{0}$, say $\mu^{0}=0.001$, and $k=$ 0 . Then, consider the following.

Step 1. Solve the direct problem (1)-(3) with the available estimate $\mathbf{P}^{k}$ in order to obtain the vector $\mathbf{U}\left(\mathbf{P}^{k}\right)=$ $\left[u\left(x_{0}, t_{1}, \mathbf{P}^{k}\right), u\left(x_{0}, t_{2}, \mathbf{P}^{k}\right), \ldots, u\left(x_{0}, t_{n}, \mathbf{P}^{k}\right)\right]$.

Step 2. Compute $F\left(\mathbf{P}^{k}\right)$ from (7).

Step 3. Compute the sensitivity matrix $\mathbf{J}^{k}$ from (11) and then the matrix $\Omega^{k}$ from (21), by using the current value of $\mathbf{P}^{k}$.

Step 4. Solve the following linear system of algebraic equations, obtained from (17).

$\left[\left(\mathbf{J}^{k}\right)^{T} \mathbf{J}^{k}+\mu^{k} \Omega^{k}\right] \Delta \mathbf{P}^{k}=\left(\mathbf{J}^{k}\right)^{T}\left[\mathbf{G}-\mathbf{U}\left(\mathbf{P}^{k}\right)\right]$ in order to compute $\Delta \mathbf{P}^{k}=\mathbf{P}^{k+1}-\mathbf{P}^{k}$.

Step 5. Compute the new estimate $\mathbf{P}^{k+1}$ as $\mathbf{P}^{k+1}=\mathbf{P}^{k}+\Delta \mathbf{P}^{k}$.

Step 6. Solve the exact problem (1)-(3) with the new estimate $\mathbf{P}^{k+1}$ in order to find $U\left(\mathbf{P}^{k+1}\right)$. Then compute $F\left(\mathbf{P}^{k+1}\right)$.

Step 7. If $F\left(\mathbf{P}^{k+1}\right) \geq F\left(\mathbf{P}^{k}\right)$, replace $\mu^{k}$ by $10 \mu^{k}$ and return to Step 4.

Step 8. If $F\left(\mathbf{P}^{k+1}\right) \leq F\left(\mathbf{P}^{k}\right)$, accept the new estimate $\mathbf{P}^{k+1}$ and emplace $\mu^{k}$ by $0.1 \mu^{k}$. 
TABLE 1: Performance of the algorithm when it is run to solve the model using four different parameters guesses.

\begin{tabular}{|c|c|c|c|c|}
\hline Starting point & 0.5 & 1 & 10 & 50 \\
\hline \multirow{3}{*}{ Iteration 12} & 0.999729028233135 & 0.999729028233183 & 0.999729028233194 & 0.999729028307261 \\
\hline & 0.499885876453067 & 0.499885876453056 & 0.499885876453057 & 0.499885876454169 \\
\hline & 0.252009862457275 & 0.252009862457315 & 0.252009862457325 & 0.25200986249336 \\
\hline Error $F$ & $8.7564944405 \times 10^{-14}$ & $8.7564944427 \times 10^{-14}$ & $8.7564944420 \times 10^{-14}$ & $8.7564944420 \times 10^{-14}$ \\
\hline
\end{tabular}

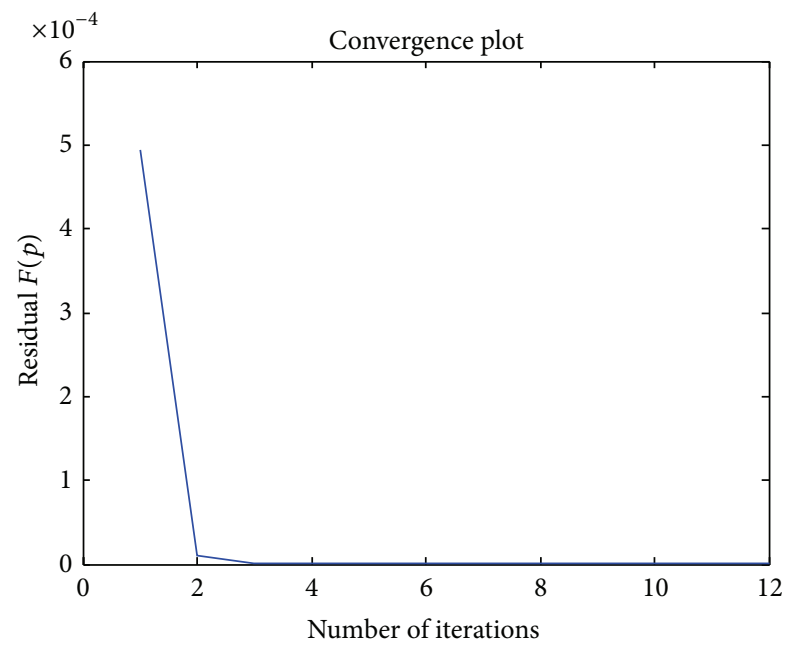

FIgURE 1: All the initial values for the parameters are set to 0.5 .

Step 9. Check the stopping criteria given by (18). Stop the iterative procedure if any of them is satisfied; otherwise, replace $k$ by $k+1$ and return to Step 3 .

\section{Calculation of Sensitivity Coefficients}

Generally, there have been two approaches for determining the gradient; the first is a discretize-then-differentiate approach and the second is a differentiate-then-discretize approach.

The first approach is to approximate the gradient of the functional by a finite difference quotient approximation, but, in general, we cannot determine the sensitivities exactly, so this method may lead to larger error.

Here, we intend to use differentiate-then-discretize approach which we refer to as the sensitivity equation method. This method can be determined more efficiently with the help of the sensitivities

$$
u_{k}=\frac{\partial u}{\partial p_{k}}, \quad k=1,2, \ldots, m+1
$$

We first differentiate the flow system (1)-(3) with respect to each of the design parameters $\left[p_{1}, p_{2}, \ldots, p_{m+1}\right]$, to obtain the $m+1$ continuous sensitivity systems: for $k=1,2, \ldots, m+1$

$$
\begin{gathered}
\frac{\partial u_{k}}{\partial t}=\frac{\partial}{\partial x}\left[\left(p_{1}+p_{2} x+\cdots+p_{m+1} x^{m}\right) \frac{\partial u_{k}}{\partial x}+x^{k-1} \frac{\partial u}{\partial x}\right], \\
u_{k}(x, 0)=0
\end{gathered}
$$

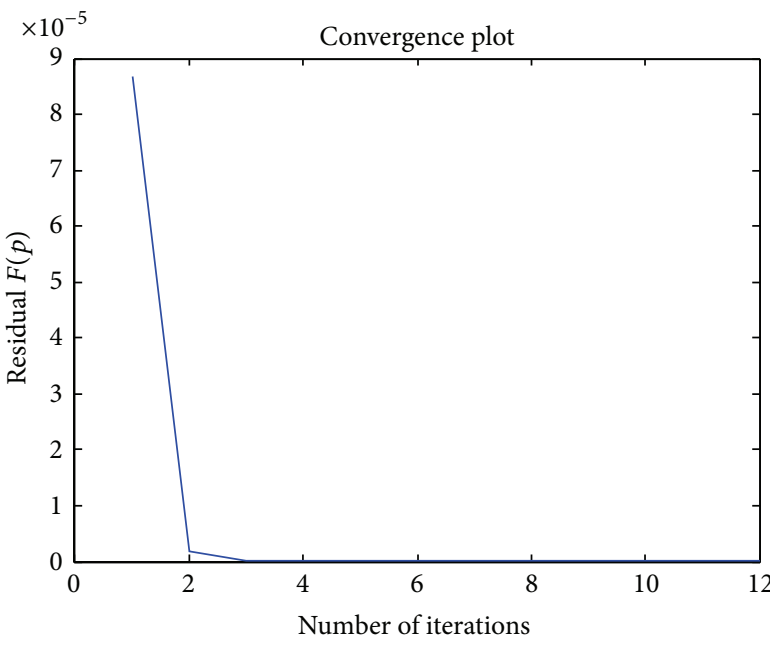

FIGURE 2: All the initial values for the parameters are set to 1.

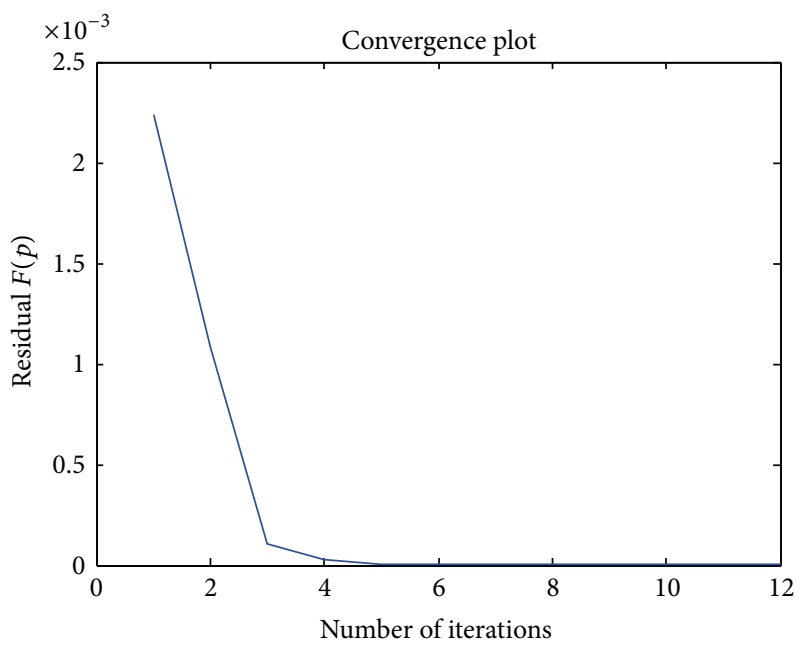

FIgURE 3: All the initial values for the parameters are set to 10.

$$
\begin{aligned}
& u_{k}(0, t)=0 \\
& u_{k}(L, t)=0
\end{aligned}
$$

There are $(m+2)$ equations; we can make them in one system equation and use the finite element methods to solve 


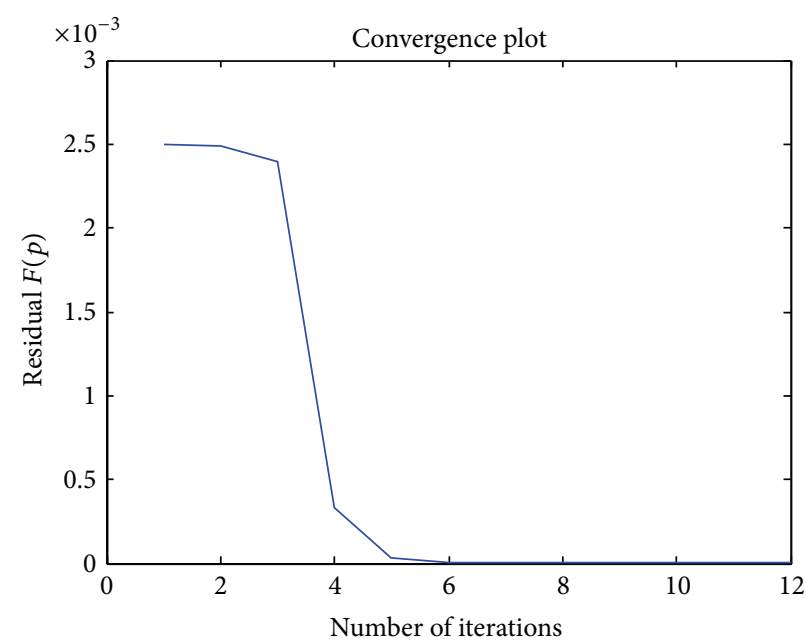

FIgURE 4: All the initial values for the parameters are set to 50.

the system of equation. Here, we give the vector form of the equation as follows:

$$
(\mathbf{P} 1)\left\{\begin{array}{l}
\frac{\partial \overrightarrow{\mathbf{U}}}{\partial t}+\nabla \cdot \overrightarrow{\mathbf{\Gamma}}=\overrightarrow{\mathbf{F}} \\
\overrightarrow{\mathbf{U}}(x, 0)=\overrightarrow{\mathbf{U}}_{0}(x) \\
\overrightarrow{\mathbf{U}}(0, t)=\overrightarrow{\mathbf{G}}_{1}(t) \\
\overrightarrow{\mathbf{U}}(L, t)=\overrightarrow{\mathbf{G}}_{2}(t),
\end{array}\right.
$$

where

$$
\overrightarrow{\boldsymbol{\Gamma}}=\left[\begin{array}{c}
\overrightarrow{\mathbf{U}}=\left[\begin{array}{c}
u \\
u_{1} \\
u_{2} \\
\vdots \\
u_{m+1}
\end{array}\right], \\
-\left(p_{1}+p_{2} x+\cdots+p_{m+1} x^{m}\right) \frac{\partial u_{2}}{\partial x}-x\left(\frac{\partial u}{\partial x}\right) \\
\vdots \\
-\left(p_{1}+p_{2} x+\cdots+p_{m+1} x^{m}\right) \frac{\partial u}{\partial x} \\
-\left(p_{1}+p_{2} x+\cdots+p_{m+1} x^{m}\right) \frac{\partial u_{m+1}}{\partial x}-\left(x^{m} \frac{\partial u}{\partial x}\right) \\
\overrightarrow{\mathbf{F}}=\left[\begin{array}{c}
f(x, t) \\
0 \\
0 \\
\vdots \\
0
\end{array}\right], \\
\overrightarrow{\mathbf{U}}_{0}(x)=\left[\begin{array}{c}
\left.u_{0}(x), 0,0, \ldots, 0\right]^{T} \\
u_{1}
\end{array}\right]
\end{array}\right.
$$

$$
\begin{aligned}
\overrightarrow{\mathbf{G}}_{1}(t) & =\left[g_{1}(t), 0,0, \ldots, 0\right]^{T}, \\
\overrightarrow{\mathbf{G}}_{2}(t) & =\left[g_{2}(t), 0,0, \ldots, 0\right]^{T} .
\end{aligned}
$$

We use the Galerkin finite element method approximation for discretizing problem (24). For this, we multiply (24) by a test function $v:[0, L] \rightarrow R, v \in V_{0}:=H_{0}^{1}(0, L)$ and integrate the obtained equation in space form 0 to $L$. We obtain the following equation:

$$
\begin{gathered}
\int_{0}^{L} \frac{\partial \mathbf{U}(x, t)}{\partial t} \\
\cdot v(x) d x-\int_{0}^{L} \nabla \boldsymbol{\Gamma} \cdot v(x) d x \\
=\int_{0}^{L} \mathbf{F}(x, t) \cdot v(x) d x ;
\end{gathered}
$$

integrating by parts gives

$$
\int_{0}^{L} \nabla \boldsymbol{\Gamma} \cdot v(x) d x=\left.(\boldsymbol{\Gamma} \cdot v(x))\right|_{0} ^{L}-\int_{0}^{L} \boldsymbol{\Gamma} \cdot \frac{\partial v(x, t)}{\partial x} d x
$$

We can change the first derivative in time and the integral. We have $v(0)=0=v(L)$, because $v \in V_{0}$. This leads to an equivalent problem (P1): $\forall t>0$, find $\mathbf{U}(x, t)$ satisfying

$$
\begin{aligned}
\frac{d}{d t} \int_{0}^{L} \mathbf{U}(x, t) & \cdot v(x) d x+\int_{0}^{L} \boldsymbol{\Gamma} \cdot \frac{\partial v(x, t)}{\partial x} d x \\
& =\int_{0}^{L} \mathbf{F}(x, t) \cdot v(x) d x
\end{aligned}
$$

for all $v \in V_{0}:=H_{0}^{1}(0, L)$. To simplify the notation, we use the scalar product in $L^{2}(0, L)$ :

$$
(f, g)=\int_{0}^{L} f(x) \cdot g(x) d x .
$$

We also can define the following bilinear form. 


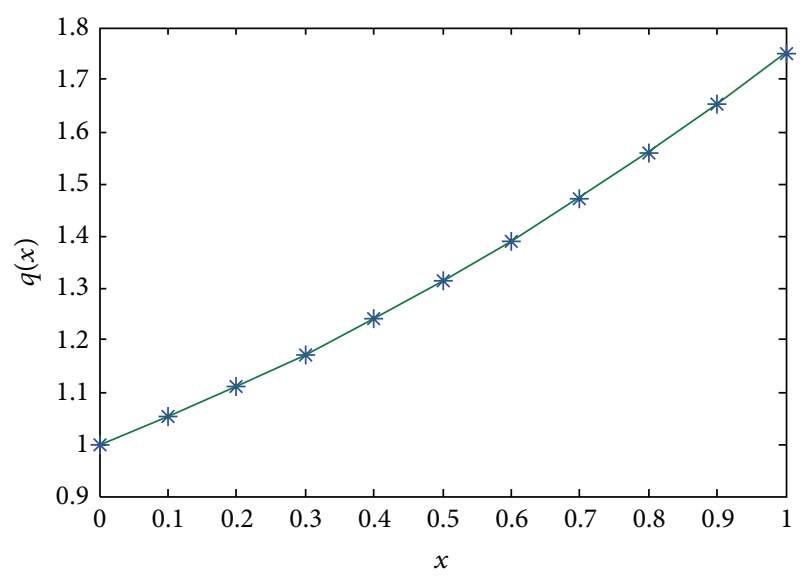

* Numerical simulation

_ True solution

FIGURE 5: The comparison chart with all the initial values for the parameters being set to 0.5 .

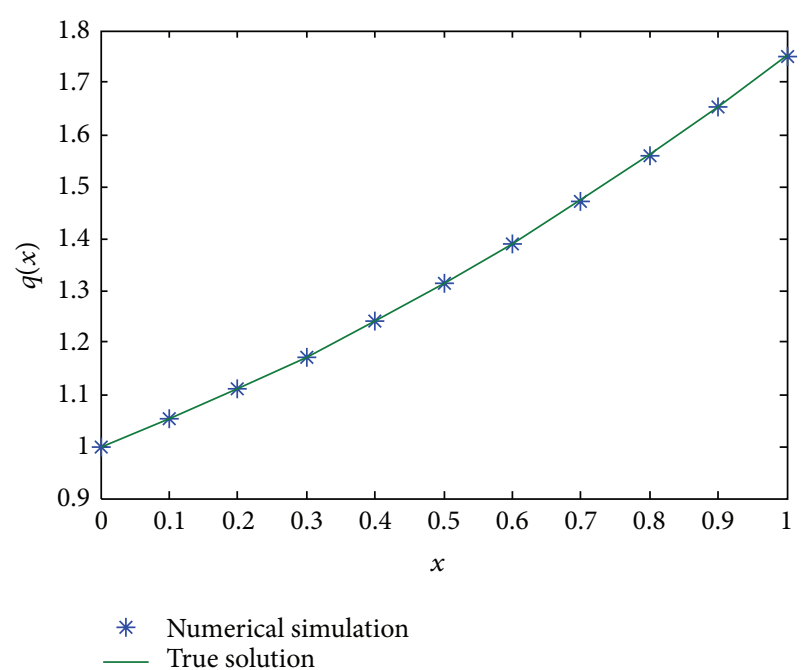

FIGURE 6: The comparison chart with all the initial values for the parameters being set to 1 .

$$
a(\mathbf{U}, v)=\int_{0}^{L} \Gamma \cdot \frac{\partial v(x, t)}{\partial x} d x=\left\{\begin{array}{l}
\int_{0}^{L}-\left(p_{1}+p_{2} x+\cdots+p_{m+1} x^{m}\right) \frac{\partial u}{\partial x} \frac{\partial v}{\partial x} d x \\
\int_{0}^{L}\left(-\left(p_{1}+p_{2} x+\cdots+p_{m+1} x^{m}\right) \frac{\partial u_{1}}{\partial x} \frac{\partial v}{\partial x}-\frac{\partial u}{\partial x} \frac{\partial v}{\partial x}\right) d x \\
\int_{0}^{L}\left(-\left(p_{1}+p_{2} x+\cdots+p_{m+1} x^{m}\right) \frac{\partial u_{2}}{\partial x} \frac{\partial v}{\partial x}-x \frac{\partial u}{\partial x} \frac{\partial v}{\partial x}\right) d x \\
\vdots \\
\int_{0}^{L}\left(-\left(p_{1}+p_{2} x+\cdots+p_{m+1} x^{m}\right) \frac{\partial u_{m+1}}{\partial x} \frac{\partial v}{\partial x}-x^{m+1} \frac{\partial u}{\partial x} \frac{\partial v}{\partial x}\right) d x
\end{array}\right.
$$

Finally, we obtain with this notation the weak problem of (P1):

$$
(\mathbf{P} 2)\left\{\begin{array}{l}
\frac{d}{d t}(\mathbf{U}, v)_{L^{2}}+a(\mathbf{U}, v)=(\mathbf{F}, v)_{L^{2}} \\
\mathbf{U}(x, 0)=\mathbf{U}_{0}(x) \\
\mathbf{U}(0, t)=\mathbf{G}_{1}(t) \\
\mathbf{U}(L, t)=\mathbf{G}_{2}(t) .
\end{array}\right.
$$

4.1. Space Discretization with the Galerkin Method. In this section, we search a semidiscrete approximation of the weak problem (P2), using the Galerkin finite element method. This leads to a first-order Cauchy problem in time.

Let $V_{h}$ be an $N_{x}+1$-dimensional subspace of $V$ and $V_{0, h}=$ $V_{h} \cap V_{0}$. Then, the following problem is an approximation of the weak problem; find $u_{h}, u_{1, h}, u_{2, h}, \ldots, u_{m+1 \cdot h} \in V_{h}$ that satisfies

$$
\begin{gathered}
\frac{d}{d t}\left(\mathbf{U}_{h}, v_{h}\right)+a\left(\mathbf{U}_{h}, v_{h}\right)=\left(\mathbf{F}, v_{h}\right), \\
\mathbf{U}_{h}(x, 0)=\mathbf{U}_{0, h}(x),
\end{gathered}
$$

$$
\begin{aligned}
& \mathbf{U}_{h}(0, t)=\mathbf{G}_{1}(t), \\
& \mathbf{U}_{h}(L, t)=\mathbf{G}_{2}(t),
\end{aligned}
$$

for all $v_{h} \in V_{0, h}$, where $\mathbf{U}_{h}=\left[u_{h}, u_{1, h}, u_{2, h}, \ldots, u_{m+1 \cdot h}\right]^{T}$.

The choice of $V_{h}$ is completely arbitrary. So, we can choose it the way that, for later treatment, it will be as easy as possible. For example, we subdivide the interval $[0, L]$ into partitions of equal distances $h$ :

$$
\begin{gathered}
0=a_{1}<a_{2}<\cdots<a_{N_{x}}<a_{N_{x}+1}=L, \\
a_{i}=(i-1) \cdot h, \\
V_{h}=\left\{v_{h} \in C^{0}[0, L]:\left.v_{h}\right|_{\left[a_{i}, a_{i+1}\right]} \in \mathbf{P}_{1}, \forall i=1, \ldots, N_{x}\right\}, \\
V_{0, h}=\left\{v_{h} \in V_{h}: v_{h}(0)=v_{h}(L)=0\right\} .
\end{gathered}
$$

Note that the finite dimension allows us to build a finite base for the corresponding space. In the case of $V_{0, h}$, we have $\left\{\varphi_{i}\right\}_{i=2}^{N_{x}}$, where $\forall i=2, \ldots, N_{x}$. 
TABLE 2: The values of $q(j \Delta x)$ and $u(j \Delta x, 0.5)$ in $x=j \Delta x$ with all the initial values being set to 1 .

\begin{tabular}{lcccr}
\hline$j$ & Numerical & Exact & Numerical & Exact \\
$(j \Delta x)$ & $u(j \Delta x, 0.5)$ & 0 \\
\hline 0 & 0.999729028233183 & 1 & 0 & 0.0605593190239173 \\
1 & 1.05223771450306 & 1.0525 & 0.120511797611786 & 0.0605520280601669 \\
2 & 1.10978659802209 & 1.11 & 0.179257059078521 & 0.120499040271796 \\
3 & 1.17237567879026 & 1.1725 & 0.236207449080344 & 0.179242065904716 \\
4 & 1.24000495680758 & 1.24 & 0.290793943250869 & 0.236194164064666 \\
5 & 1.31267443207404 & 1.3125 & 0.342471828361625 & 0.290786288212692 \\
6 & 1.39038410458965 & 1.39 & 0.390726114897089 & 0.342472971890064 \\
7 & 1.47313397435441 & 1.4725 & 0.435076630410587 & 0.390737778838824 \\
8 & 1.56092404136831 & 1.56 & 0.475082717530532 & 0.435098463062163 \\
9 & 1.65375430563136 & 1.6525 & 0.510347406713368 & 0.475111787267016 \\
10 & 1.75162476714355 & 1.75 & 0.510377951544573 \\
\hline
\end{tabular}

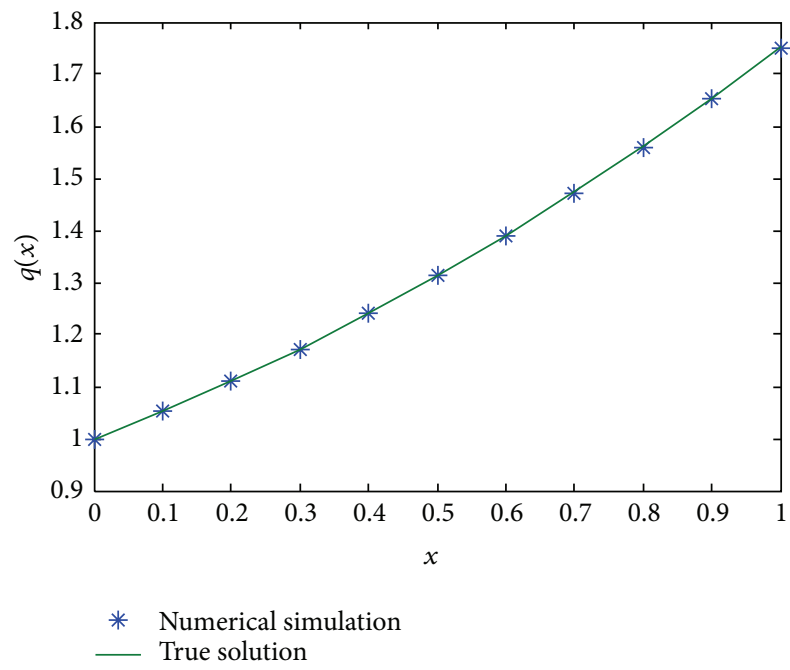

Figure 7: The comparison chart with all the initial values for the parameters being set to 10 .

$$
\begin{aligned}
& \text { Consider } \\
& \varphi_{i}(x)= \begin{cases}0 & x \in\left[a_{0}, a_{i-1}\right] \\
\frac{x}{h}-(i-2) & x \in\left[a_{i-1}, a_{i}\right] \\
i-\frac{x}{h} & x \in\left[a_{i}, a_{i+1}\right] \\
0 & x \in\left[a_{i+1}, a_{N_{x}+1}\right],\end{cases}
\end{aligned}
$$

while we add for $V_{h}$ the two functions $\varphi_{1}$ and $\varphi_{N_{x}+1}$ defined as

$$
\begin{gathered}
\varphi_{1}(x)= \begin{cases}1-\frac{x}{h}, & \text { if } x \in\left[a_{1}, a_{2}\right] \\
0, & \text { if } x \in\left[a_{2}, a_{N_{x}+1}\right],\end{cases} \\
\varphi_{N_{x}+1}(x)= \begin{cases}0, & \text { if } x \in\left[a_{1}, a_{N_{x}}\right] \\
\frac{x}{h}-N_{x}+1, & \text { if } x \in\left[a_{N_{x}}, a_{N_{x}+1}\right],\end{cases}
\end{gathered}
$$

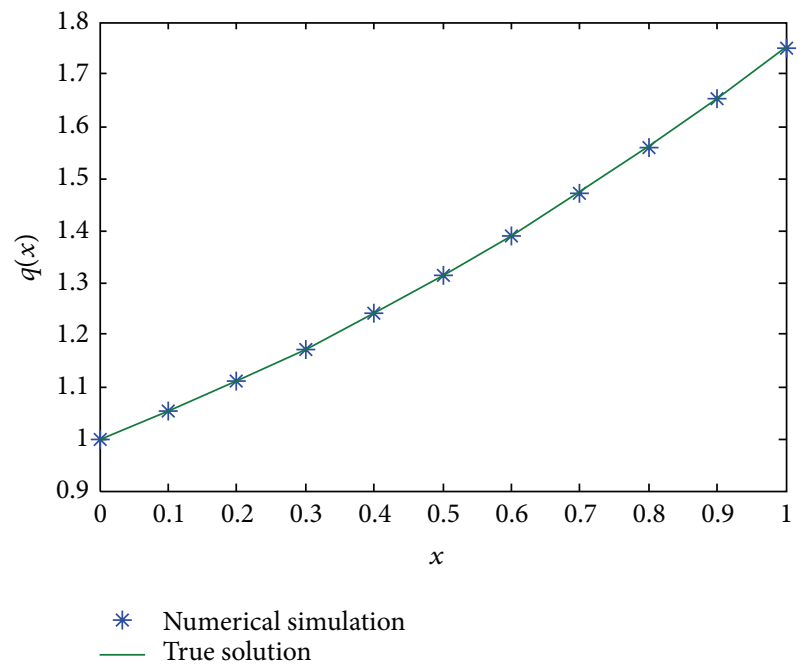

FIGURE 8: The comparison chart with all the initial values for the parameters being set to 50 .

so that we can write $\mathbf{U}_{h}$ as a linear combination of the basic elements:

$$
\begin{aligned}
\mathbf{U}_{h}(x, t) & =\sum_{j=1}^{N_{x}+1} \widetilde{\mathbf{U}}_{j}(t) \cdot \varphi_{j}(x), \\
\mathbf{U}_{0, h}(x, t) & =\sum_{j=1}^{N_{x}+1} \mathbf{U}_{0}\left(x_{j}\right) \cdot \varphi_{j}(x),
\end{aligned}
$$

where $\widetilde{\mathbf{U}}_{1}(t)=\mathbf{G}_{0}(t)$ and $\widetilde{\mathbf{U}}_{N_{x}+1}(t)=\mathbf{G}_{1}(t)$. Knowing that $a(\cdot, \cdot)$ is bilinear form and that $(32)$ is valid for each element of the base $\left\{\varphi_{i}\right\}_{i=2}^{N_{x}}$, we obtain

$$
\begin{array}{r}
\sum_{j=1}^{N_{x}+1} \frac{d}{d t} \widetilde{\mathbf{U}}_{j}(t) \cdot\left(\varphi_{j}, \varphi_{i}\right) \\
+\sum_{j=1}^{N_{x}+1} \widetilde{\mathbf{U}}_{j}(t) \cdot a\left(\varphi_{j}, \varphi_{i}\right)=\left(\mathbf{F}, \varphi_{i}\right), \\
\forall i=2, \ldots, N_{x} .
\end{array}
$$




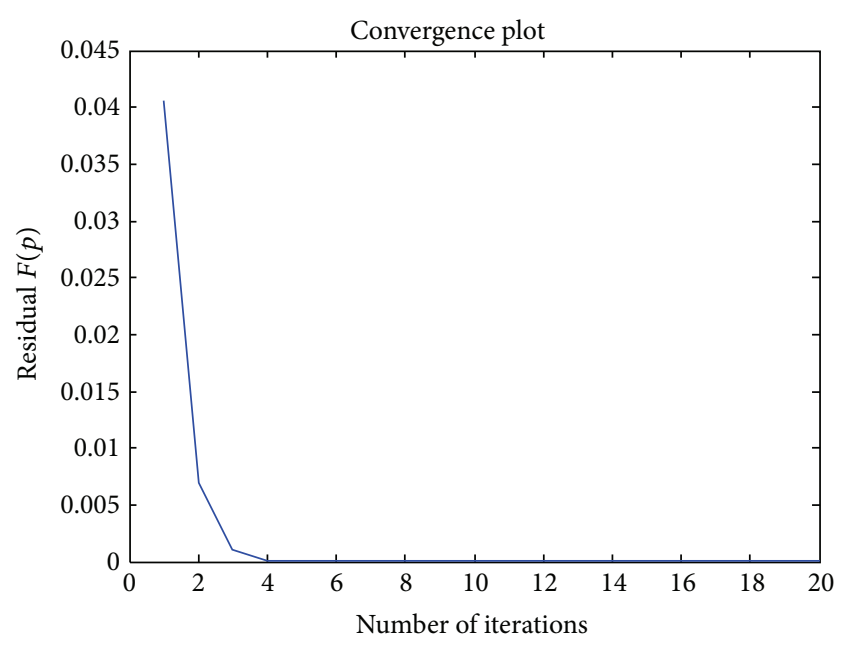

Figure 9: All the initial values for the parameters are set to 0.1 .

This equation can be written in a vector form. For this, we define the vectors $\overrightarrow{\mathbf{u}}$, $\overrightarrow{\mathbf{u}}_{0}$, and $\overrightarrow{\mathbf{F}}$ with components

$$
\mathbf{F}_{i}(t)=\left(\mathbf{F}, \varphi_{i}\right)_{L^{2}}, \quad \mathbf{u}_{j}(t):=\widetilde{\mathbf{u}}_{j}(t), \quad \mathbf{u}_{0, j}=\mathbf{u}_{0}\left(\mathbf{x}_{j}\right),
$$

and matrices $\mathbf{M}$ and $\mathbf{A}$ as

$$
m_{i j}:=\left(\varphi_{i}, \varphi_{j}\right)_{L^{2}}, \quad a_{i j}:=a\left(\varphi_{i}, \varphi_{j}\right) .
$$

Note that $\mathbf{M}, \mathbf{A} \in \mathbf{R}^{N_{x}-1 \times N_{x}+1}, \overrightarrow{\mathbf{u}} \in \mathbf{R}^{N_{x}+1}$, and $\overrightarrow{\mathbf{F}} \in \mathbf{R}^{N_{x}-1}$. So that (37) is equal to the Cauchy problem

$$
\begin{gathered}
\mathbf{M} \frac{d}{d x} \overrightarrow{\mathbf{u}}(t)+\mathbf{A} \cdot \overrightarrow{\mathbf{u}}(t)=\overrightarrow{\mathbf{F}}(t), \\
\overrightarrow{\mathbf{u}}\left(t_{0}\right)=\overrightarrow{\mathbf{u}}_{\mathbf{0}},
\end{gathered}
$$

the Crank-Nicolson method can be applied to (40) at time $t_{k}$, resulting in

$$
\mathbf{M} \cdot\left(\frac{\overrightarrow{\mathbf{u}}_{k+1}-\overrightarrow{\mathbf{u}}_{k}}{\Delta t}\right)+\frac{1}{2} \mathbf{A} \cdot \overrightarrow{\mathbf{u}}_{k+1}+\frac{1}{2} \mathbf{A} \cdot \overrightarrow{\mathbf{u}}_{k}=\frac{1}{2}\left(\overrightarrow{\mathbf{F}}_{k}+\overrightarrow{\mathbf{F}}_{k+1}\right),
$$

where $\overrightarrow{\mathbf{u}}_{k}=\overrightarrow{\mathbf{u}}\left(t_{k}\right), \overrightarrow{\mathbf{F}}_{k}=\overrightarrow{\mathbf{F}}\left(t_{k}\right), k=0,1, \ldots$

Equation (41) can be written in simple form as

$$
\left(\mathbf{M}+\frac{\Delta t}{2} \mathbf{A}\right) \cdot \overrightarrow{\mathbf{u}}_{k+1}=\left(\mathbf{M}-\frac{\Delta t}{2} \mathbf{A}\right) \cdot \overrightarrow{\mathbf{u}}_{k}+\frac{\Delta t}{2}\left(\overrightarrow{\mathbf{F}}_{k}+\overrightarrow{\mathbf{F}}_{k+1}\right) .
$$

The algebraic system (42) is solved by Gauss elimination method.

\section{Numerical Experiment}

In this section, we are going to demonstrate some numerical results for $(u(x, t), q(x))$ in the inverse problem (1)-(4). Therefore, the following examples are considered and the solutions are obtained.

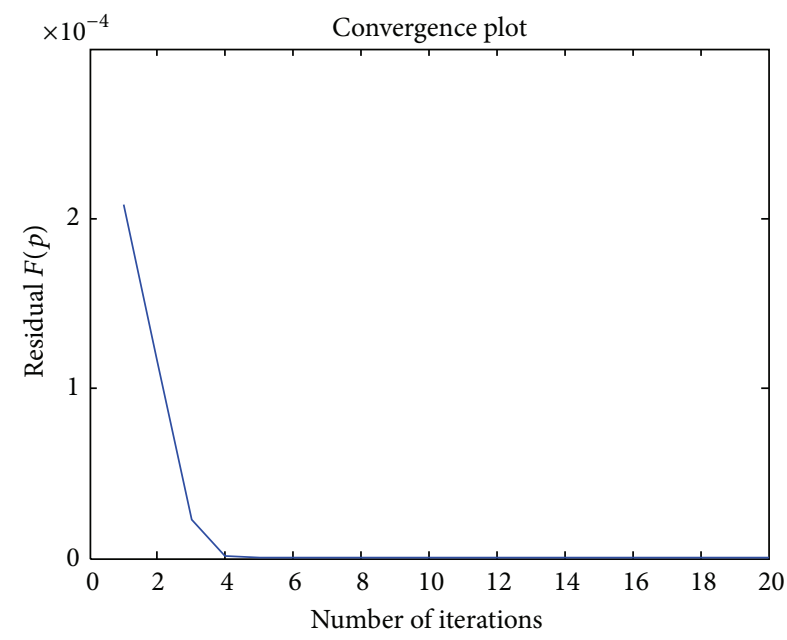

Figure 10: All the initial values for the parameters are set to 0.5 .

Example 1. Consider (1)-(3) with

$$
\begin{gathered}
u(x, 0)=\sin x, \quad 0 \leq x \leq 1, \\
u(0, t)=0, \quad 0 \leq t \leq 1, \\
u(1, t)=\sin (1) e^{-t}, \quad 0 \leq t \leq 1, \\
f(x, t)=\left(\sin x\left(\frac{x^{2}}{4}+\frac{x}{2}+1\right)\right) e^{-t} \\
-\frac{(x+1)}{2} \cos (x) e^{-t}-\sin x e^{-t}, \\
0 \leq x \leq 1, \quad 0 \leq t \leq 1 .
\end{gathered}
$$

We obtain the unique exact solution

$$
\begin{gathered}
q(x)=1+0.5 x+0.25 x^{2}, \\
u(x, t)=\sin (x) e^{-t} .
\end{gathered}
$$

We take the observed data $g$ as

$$
g(t)=u(0.5, t)=\sin (0.5) e^{-t}, \quad 0 \leq t \leq 1 .
$$

The unknown function $q(x)$ is defined as the following form:

$$
\hat{q}(x)=p_{1}+p_{2} x+p_{3} x^{2},
$$

where $p_{1}, p_{2}$, and $p_{3}$ are unknown coefficients.

Table 1 shows how the Levenberg-Marquardt algorithm can find the best parameters after 12 iterations when it is initialized in four different points.

Figures 1,2, 3, and 4 show the fitness of the estimated parameters and the rate of convergence.

Figures 5, 6, 7, and 8 show the comparison between the inversion results $q(x)$ and the exact value $q(x)$.

Table 2 shows the values of $q(j \Delta x)$ and $u(j \Delta x, 0.5)$ in $x=$ $j \Delta x$ with all the initial values being set to 1 . 
TABLE 3: Performance of the algorithm when it is run to solve the model using four different parameters guesses.

\begin{tabular}{|c|c|c|c|c|c|c|c|c|c|c|c|c|c|c|c|}
\hline \multirow{2}{*}{ Starting point } & 0.1 & 0.1 & 0.1 & 0.1 & 0.5 & 0.5 & 0.5 & 0.5 & 1 & 1 & 1 & 2 & 2 & 2 & 2 \\
\hline & 0.1 & 0.1 & 0.1 & 0.1 & 0.5 & 0.5 & 0.5 & 0.5 & 1 & 1 & 1 & 2 & 2 & 2 & 2 \\
\hline \multirow{8}{*}{ Iteration 20} & \multicolumn{4}{|c|}{1.01536263526644} & \multicolumn{4}{|c|}{1.01536263500695} & \multicolumn{3}{|c|}{1.01536263525763} & \multicolumn{4}{|c|}{1.01536263525905} \\
\hline & \multicolumn{4}{|c|}{0.896348846894057} & \multicolumn{4}{|c|}{0.896348850692318} & \multicolumn{3}{|c|}{0.896348847022403} & \multicolumn{4}{|c|}{0.896348846999736} \\
\hline & \multicolumn{4}{|c|}{0.954285303464511} & \multicolumn{4}{|c|}{0.954285278486704} & \multicolumn{3}{|c|}{0.954285302637587} & \multicolumn{4}{|c|}{0.954285302790922} \\
\hline & \multicolumn{4}{|c|}{-0.890298938193057} & \multicolumn{4}{|c|}{-0.890298849338373} & \multicolumn{3}{|c|}{-0.890298935334171} & \multicolumn{4}{|c|}{-0.890298935876618} \\
\hline & \multicolumn{4}{|c|}{1.40131927153131} & \multicolumn{4}{|c|}{1.40131909032315} & \multicolumn{3}{|c|}{1.40131926588117} & \multicolumn{4}{|c|}{1.40131926696099} \\
\hline & \multicolumn{4}{|c|}{-0.871276408882294} & \multicolumn{4}{|c|}{-0.871276197318301} & \multicolumn{3}{|c|}{-0.871276402487896} & \multicolumn{4}{|c|}{-0.87127640370648} \\
\hline & \multicolumn{4}{|c|}{0.183785623507722} & \multicolumn{4}{|c|}{0.183785492186491} & \multicolumn{3}{|c|}{0.18378561965322} & \multicolumn{4}{|c|}{0.183785620380814} \\
\hline & \multicolumn{4}{|c|}{0.0359103726343979} & \multicolumn{4}{|c|}{0.0359104061952515} & \multicolumn{3}{|c|}{0.0359103735933848} & \multicolumn{4}{|c|}{0.0359103734148875} \\
\hline Error $F$ & \multicolumn{4}{|c|}{$7.89749200363512 \times 10^{-11}$} & \multicolumn{4}{|c|}{$7.89749200363504 \times 10^{-11}$} & 7.897492003 & -20 & $38 \times 10^{-11}$ & 7.897492 & 003 & 538 & $\times 10^{-11}$ \\
\hline
\end{tabular}

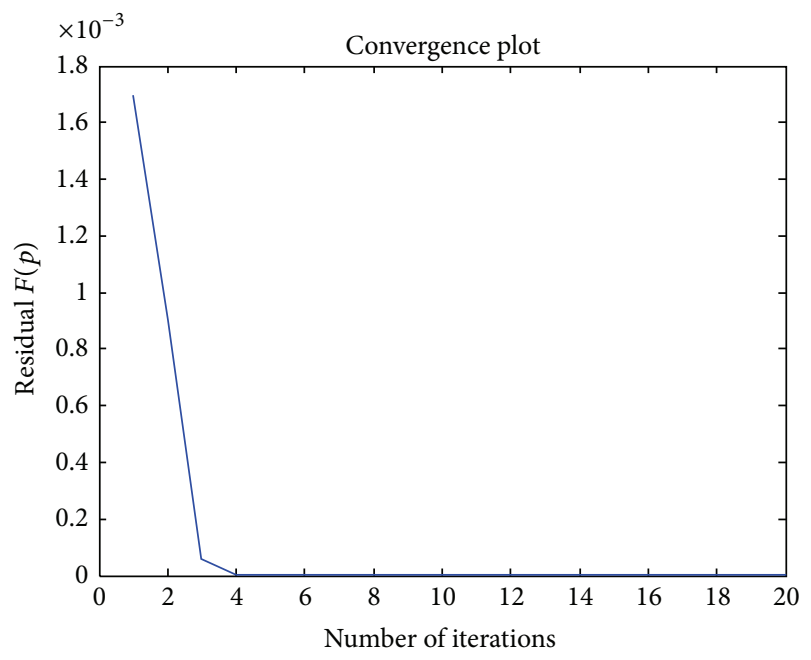

Figure 11: All the initial values for the parameters are set to 1.

Example 2. Consider (1)-(3) with

$$
\begin{gathered}
u(x, 0)=x e^{-x}, \quad 0 \leq x \leq 1, \\
u(0, t)=t e^{-t}, \quad 0 \leq t \leq 1, \\
u(1, t)=(1+t) e^{-1-t}, \quad 0 \leq t \leq 1, \\
f(x, t)=e^{-(t+x)}-(t+x) e^{-(t+x)} \\
-e^{x}\left(e^{-(t+x)}-(t+x) e^{-(t+x)}\right) \\
+e^{x}\left(2 e^{-(t+x)}-(t+x) e^{-(t+x)}\right) \\
0 \leq x \leq 1, \quad 0 \leq t \leq 1 .
\end{gathered}
$$

We obtain the unique exact solution

$$
\begin{gathered}
q(x)=e^{x}, \\
u(x, t)=(x+t) e^{-x-t} .
\end{gathered}
$$

We take the observed data $g$ as

$$
g(t)=u(0.5, t)=(0.5+t) e^{(-0.5-t)}, \quad 0 \leq t \leq 1 .
$$

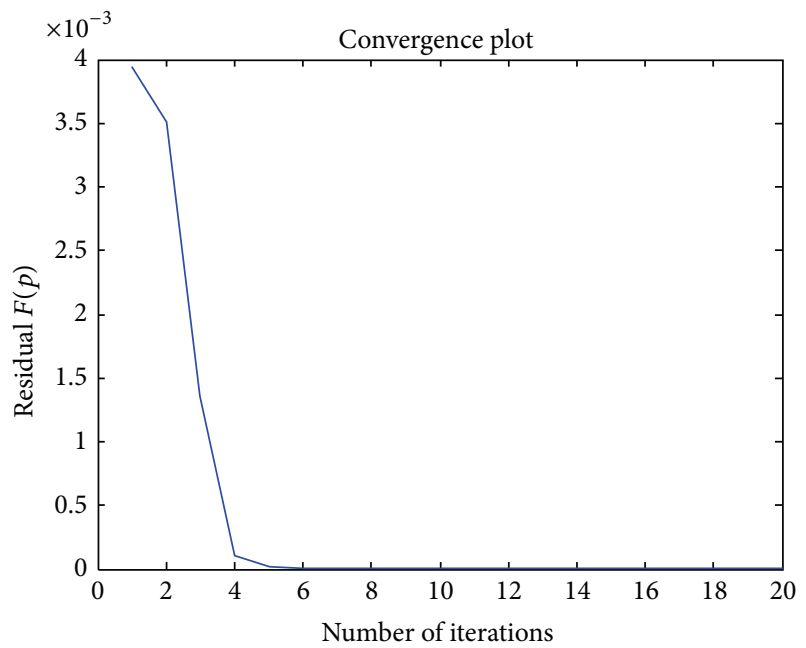

Figure 12: All the initial values for the parameters are set to 2 .

The unknown function $q(x)$ is defined as the following form.

$$
q \hat{(x)}=p_{1}+p_{2} x+p_{3} x^{2}+p_{4} x^{3}+p_{5} x^{4}+p_{6} x^{5}+p_{7} x^{6}, \text { where }
$$
$p_{1}, p_{2}, \ldots, p_{7}$ are unknown coefficients.

Table 3 shows how the Levenberg-Marquardt algorithm can find the best parameters after 20 iterations when it is initialized in four different points.

Figures 9, 10, 11, and 12 show the fitness of the estimated parameters and the rate of convergence.

Figures 13, 14, 15, and 16 show the comparison between the inversion results $q(x)$ and the exact value $q(x)$.

Table 4 shows the values of $q(j \Delta x)$ and $u(j \Delta x, 0.5)$ in $x=$ $j \Delta x$ with all the initial values being set to 1 .

\section{Conclusions}

A numerical method to estimate the temperature $u(x, t)$ and the coefficient $q(x)$ is proposed for an IHCP and the following results are obtained.

(1) The present study successfully applies the numerical method involving the Levenberg-Marquardt algorithm in conjunction with the Galerkin finite element method to an IHCP. 
TABLE 4: The values of $q(j \Delta x)$ and $u(j \Delta x, 0.5)$ in $x=j \Delta x$ with all the initial values being set to 1 .

\begin{tabular}{ccccc}
\hline$j$ & Numerical & Exact & Numerical & Exact \\
$q(j \Delta x)$ & $u(j \Delta x, 0.5)$ & $u(j \Delta x, 0.5)$ \\
\hline 0 & 1.01536263525763 & 1 & 0.303342962644088 & 0.303265329856317 \\
1 & 1.11378168059013 & 1.10517091807565 & 0.329201964677126 & 0.329286981656416 \\
2 & 1.22765694959399 & 1.22140275816017 & 0.347492882224886 & 0.347609712653987 \\
3 & 1.35549021305874 & 1.349858807576 & 0.359347568702678 & 0.359463171293777 \\
4 & 1.4973722149265 & 1.49182469764127 & 0.365808711321159 & 0.365912693766539 \\
5 & 1.65432828415206 & 1.64872127070013 & 0.367792378208857 & 0.367879441171442 \\
6 & 1.82785056869393 & 1.82211880039051 & 0.366091218454182 & 0.366158192067887 \\
7 & 2.01963499046464 & 2.01375270747048 & 0.361387761473796 & 0.361433054294643 \\
8 & 2.23154102006879 & 2.22554092849247 & 0.354267869273063 & 0.354291330944216 \\
9 & 2.46579237015687 & 2.45960311115695 & 0.345233023618059 & 0.345235749518249 \\
10 & 2.72543670622333 & 2.71828182845905 & 0.334712604803175 & 0.334695240222645 \\
\hline
\end{tabular}

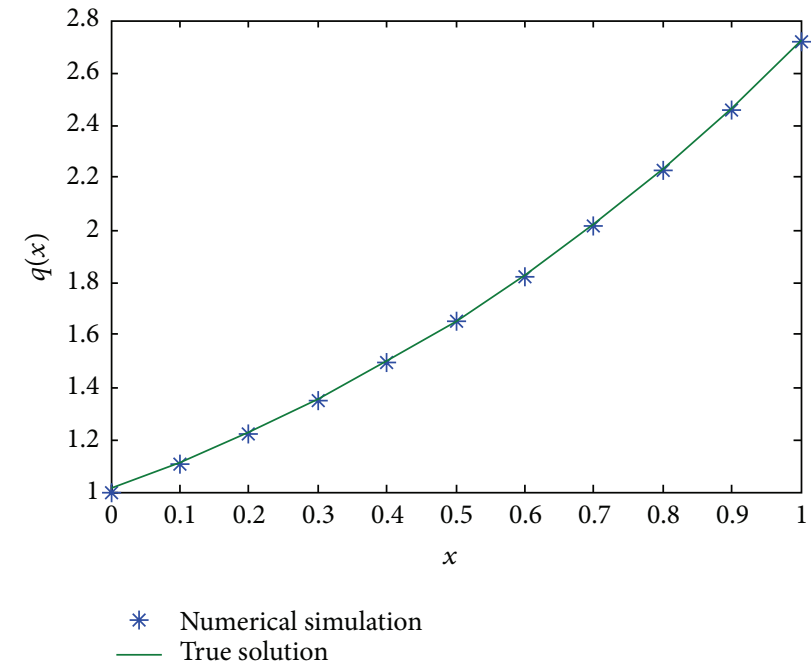

FIGURE 13: The comparison chart with all the initial values for the parameters being set to 0.1 .

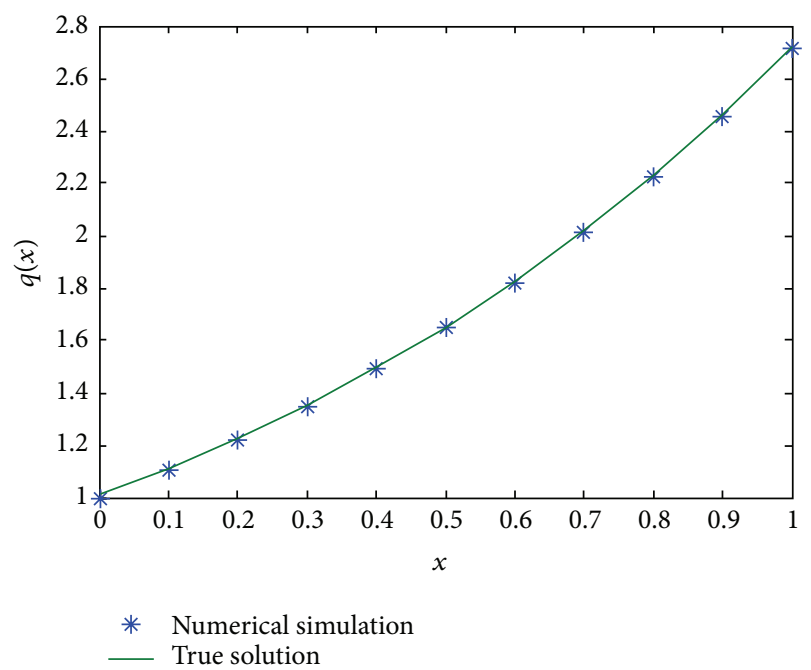

FIGURE 14: The comparison chart with all the initial values for the parameters being set to 0.5 .

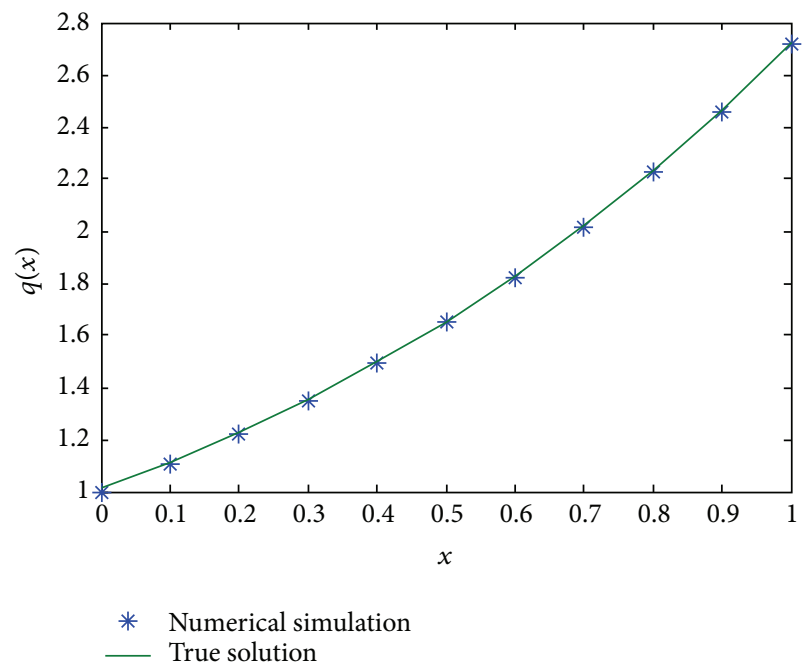

FIGURE 15: The comparison chart with all the initial values for the parameters being set to 1 .

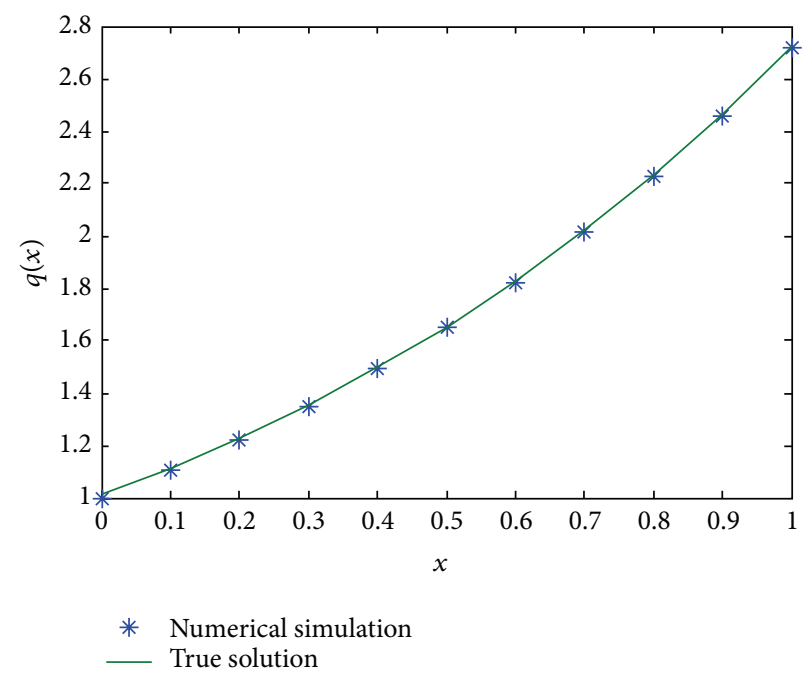

FIGURE 16: The comparison chart with all the initial values for the parameters being set to 2 . 
(2) From the illustrated example, it can be seen that the proposed numerical method is efficient and accurate to estimate the temperature $u(x, t)$ and the coefficient $q(x)$.

\section{Conflict of Interests}

The authors declare that there is no conflict of interests regarding the publication of this paper.

\section{Acknowledgments}

The work of the author is supported by the Special Funds of the National Natural Science Foundation of China (nos. 51190093 and 51179151). The author would like to thank the referees for constructive suggestions and comments.

\section{References}

[1] A. Shidfar and G. R. Karamali, "Numerical solution of inverse heat conduction problem with nonstationary measurements," Applied Mathematics and Computation, vol. 168, no. 1, pp. 540548, 2005.

[2] A. Shidfar, G. R. Karamali, and J. Damirchi, "An inverse heat conduction problem with a nonlinear source term," Nonlinear Analysis: Theory, Methods \& Applications, vol. 65, no. 3, pp. 615621, 2006.

[3] A. Shidfar and R. Pourgholi, "Numerical approximation of solution of an inverse heat conduction problem based on Legendre polynomials," Applied Mathematics and Computation, vol. 175, no. 2, pp. 1366-1374, 2006.

[4] A. Shidfar and H. Azary, "An inverse problem for a nonlinear diffusion equation," Nonlinear Analysis: Theory, Methods \& Applications, vol. 28, no. 4, pp. 589-593, 1997.

[5] K. Kurpisz and A. J. Nowak, "BEM approach to inverse heat conduction problems," Engineering Analysis with Boundary Elements, vol. 10, no. 4, pp. 291-297, 1992.

[6] H. Han, D. B. Ingham, and Y. Yuan, "The boundary element method for the solution of the backward heat conduction equation," Journal of Computational Physics, vol. 116, no. 2, pp. 292-299, 1995.

[7] J. Skorek, "Applying the least squares adjustment technique for solving inverse heat conduction problems," in Proceedings of the 8 th Conference on Numerical Methods in Laminar and Turbulent Flow, C. Taylor, Ed., pp. 189-198, Pinerdage Press, Swansea, U.K, August 1993.

[8] R. Pasquetti and C. le Niliot, "Boundary element approach for inverse heat conduction problems: application to a bidimensional transient numerical experiment," Numerical Heat Transfer B: Fundamentals, vol. 20, no. 2, pp. 169-189, 1991.

[9] D. B. Ingham and Y. Yuan, "The solution of a nonlinear inverse problem in heat transfer," IMA Journal of Applied Mathematics, vol. 50, no. 2, pp. 113-132, 1993.

[10] E. Hensel, Inverse Theory and Applications for Engineers, Prentice Hall, New Jersey, NJ, USA, 1991.

[11] M. N. Özisik, Inverse Heat Transfer: Fundamentals and Applications, Taylor \& Francis, New York, NY, USA, 2000.

[12] R. Pourgholi, N. Azizi, Y. S. Gasimov, F. Aliev, and H. K. Khalafi, "Removal of numerical instability in the solution of an inverse heat conduction problem," Communications in
Nonlinear Science and Numerical Simulation, vol. 14, no. 6, pp. 2664-2669, 2009.

[13] A. Shidfar, R. Pourgholi, and M. Ebrahimi, "A numerical method for solving of a nonlinear inverse diffusion problem," Computers \& Mathematics with Applications, vol. 52, no. 6-7, pp. 1021-1030, 2006.

[14] J. Wang and N. Zabaras, "A Bayesian inference approach to the inverse heat conduction problem," International Journal of Heat and Mass Transfer, vol. 47, no. 17-18, pp. 3927-3941, 2004.

[15] M. Dehghan, "Determination of an unknown parameter in a semi-linear parabolic equation," Mathematical Problems in Engineering, vol. 8, no. 2, pp. 111-122, 2002.

[16] J. Lihua and M. Changfeng, "On L-M method for nonlinear equations," Journal of Mathematics, vol. 29, no. 3, pp. 253-259, 2009.

[17] P. Chen, "Why not use the Levenberg-Marquardt method for fundamental matrix estimation?" The Institution of Engineering and Technology, vol. 4, no. 4, pp. 286-288, 2010. 


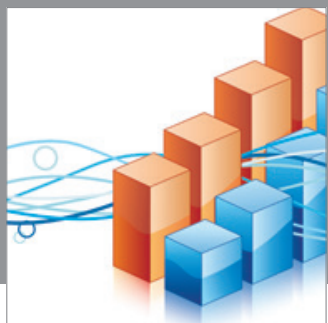

Advances in

Operations Research

mansans

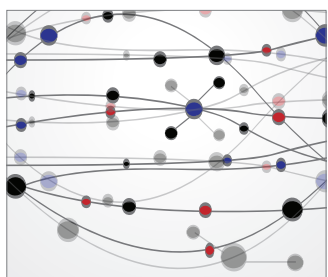

The Scientific World Journal
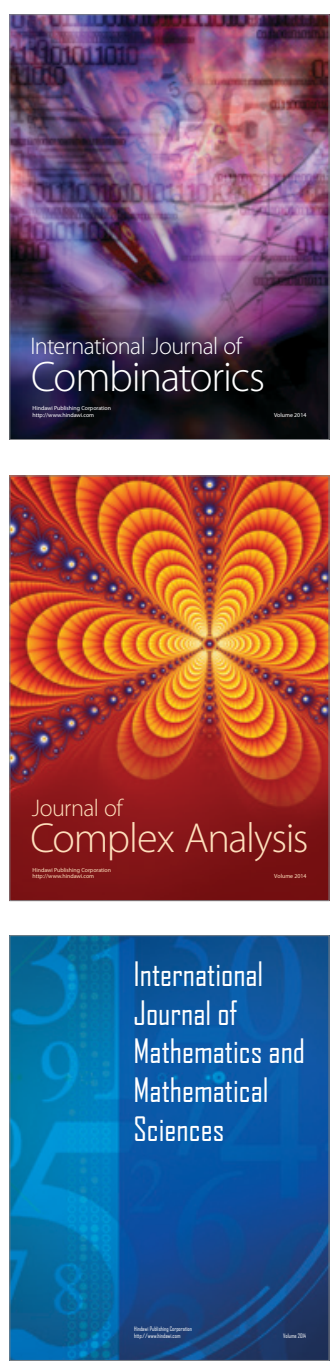
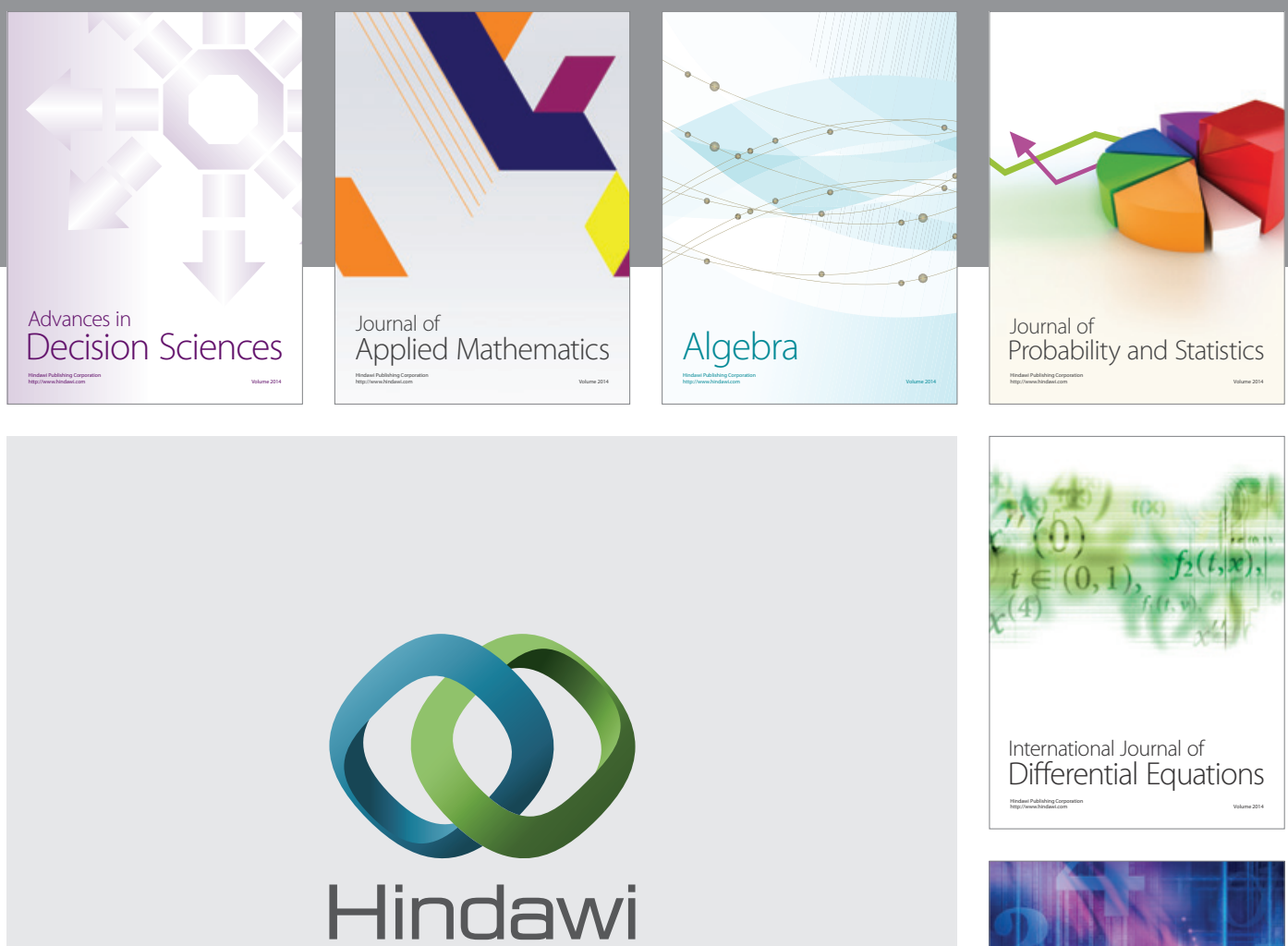

Submit your manuscripts at http://www.hindawi.com
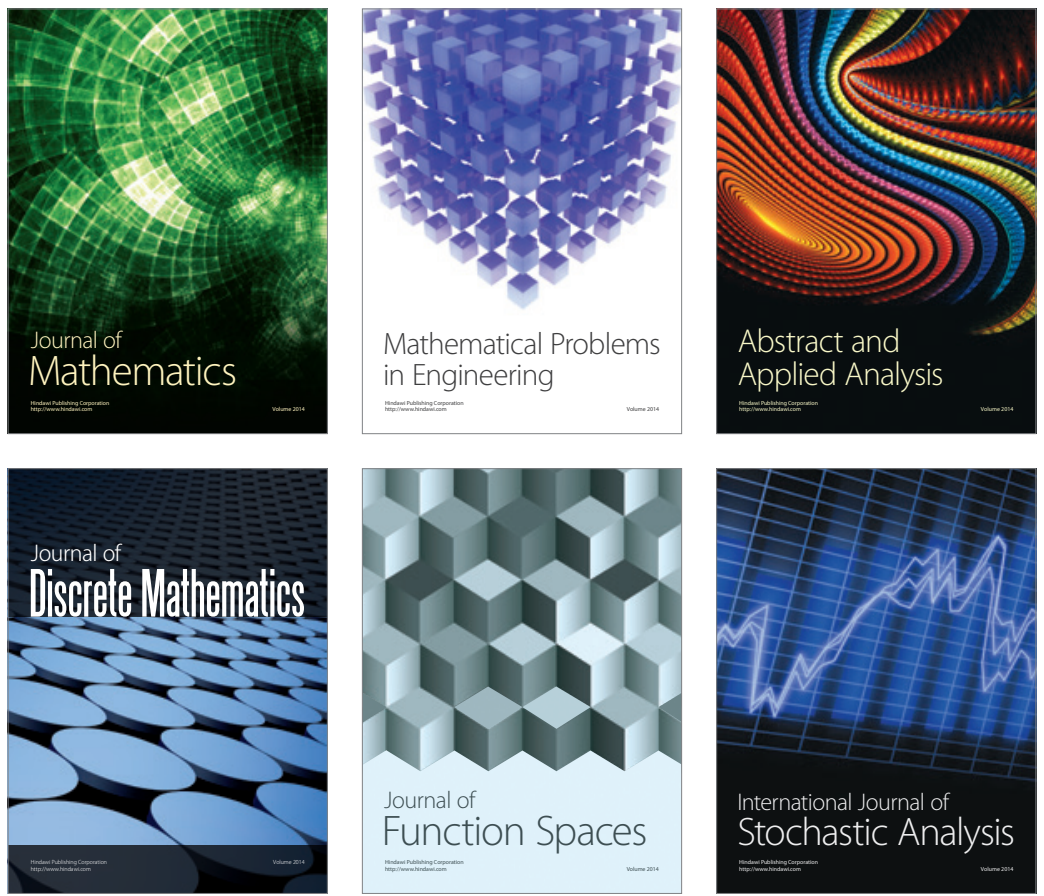

Journal of

Function Spaces

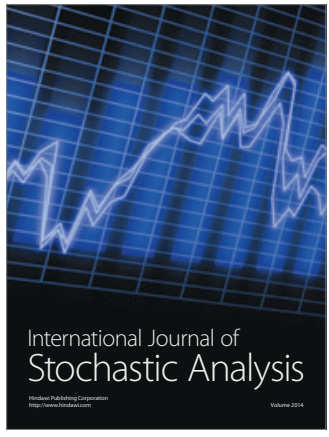

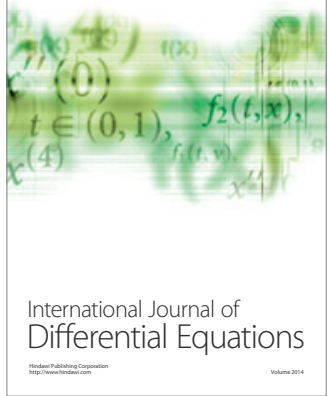
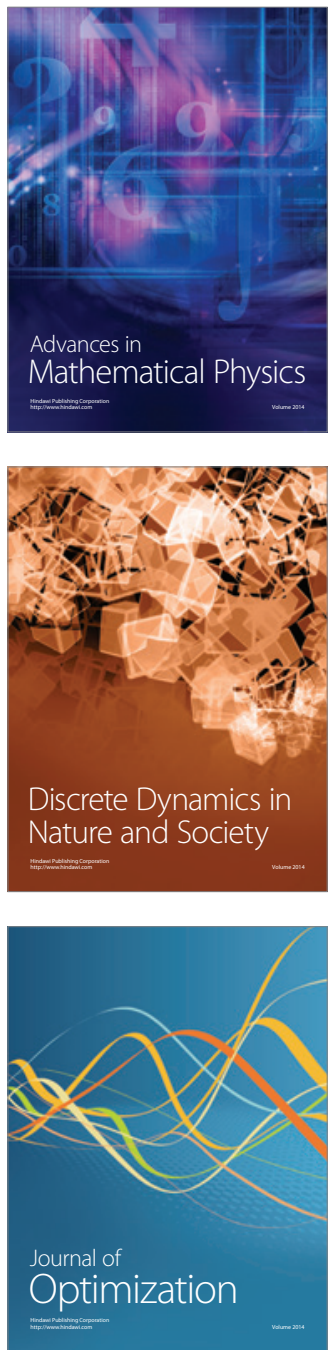